\title{
对两类带有非局部项的水波模型的半隐式谱缺陷 校正法及其衰减性质的数值研究
}

献给林群教授 80 华诞

\section{毛志平 ${ }^{1}$ ，沈捷 ${ }^{(1)}$ (2)}

(1) 厦门大学数学科学学院, 厦门 361005 ;

(2) Department of Mathematics, Purdue University, West Lafayette, IN 47907, USA

E-mail: maozhiping87@gmail.com, shen@math.purdue.edu

收稿日期: 2014-10-29；接受日期: 2015-03-02；＊通信作者

国家自然科学基金 (批准号: 91130002 和 11371298) 资助项目

摘要 本文主要对两类带非局部项水波模型的衰减性质进行数值研究, 第一类模型是具有时间非局 部黏性项的 $\mathrm{KdV}$ 型方程:

$$
u_{t}+u_{x}+\beta u_{x x x}+\frac{\sqrt{\nu}}{\sqrt{\pi}} \int_{0}^{t} \frac{u_{t}(s)}{\sqrt{t-s}} d s+u u_{x}=\nu u_{x x} ;
$$

另一类为空间非局部黏性扩散 - 色散的 Kakutani-Matsuuchi 模型:

$$
u_{t}-\beta u_{t x x}+\nu\left(D^{\frac{1}{2}}+\mathcal{F}^{-1}\left(\mathrm{i}|\zeta|^{\frac{1}{2}} \operatorname{sign}(\zeta) \widehat{u}(\zeta)\right)\right)+\gamma u u_{x}=0 .
$$

对这两类水波模型的离散，在空间上，本文采用 Fourier 变换; 在时间上, 则结合半隐式格式的计算方 便性和谱缺陷校正法的高阶性质, 采用半隐式谱缺陷校正法 (SISDC). 通过一系列的数值试验, 本文研 究模型中黏性项、几何色散项、非线性项和计算区域大小等对水波衰减性质的影响.

关键词 水波 衰减率 半隐式谱缺陷校正

MSC (2010) 主题分类 $35 \mathrm{Q} 35,35 \mathrm{Q} 53,76 \mathrm{~B} 15$

\section{1 引言}

研究黏性对水波渐近模型的影响是一项非常有挑战性的课题, 对该课题的研究也日益增多. 文 献 $[1,2]$ 分别独立地推导出了在黏性浅水区域长型重力波的渐近模型, 这些模型都是具有 Boussinesq 形式且带黏性项的. 文献 [3] 则给出了一种简化的单向波模型. 最近, 文献 [4] 同时从理论和数值上研 究了如下的一个带有非局部黏性色散项的水波模型的衰减率,

$$
u_{t}+u_{x}+\beta u_{x x x}+\frac{\sqrt{\nu}}{\sqrt{\pi}} \int_{0}^{t} \frac{u_{t}(s)}{\sqrt{t-s}} d s+\gamma u u_{x}=\alpha u_{x x},
$$


其中 $u$ 是流体的水平速率. 在该模型中, $\alpha u_{x x}$ 是黏性扩散项, $\beta u_{x x x}$ 则表示几何色散, $\frac{\sqrt{\nu}}{\sqrt{\pi}} \int_{0}^{t} \frac{u_{t}(s)}{\sqrt{t-s}} d s$ 是 描述非局部扩散 - 色散项的时间 $\frac{1}{2}$ 阶导数. $\alpha, \beta, \gamma$ 和 $\nu$ 是用来平衡黏性、色散和非线性项对模型的 影响的非负参数. Chen 等人 ${ }^{[4]}$ 给出了上述模型在 $\alpha=\nu$ 和 $\beta=0$ 下, 即

$$
u_{t}+u_{x}+\frac{\sqrt{\nu}}{\sqrt{\pi}} \int_{0}^{t} \frac{u_{t}(s)}{\sqrt{t-s}} d s+u u_{x}=\nu u_{x x}
$$

对小初始数据的一个全局存在性及其衰减率的结论. 现将结论通过下面定理 1 给出.

定理 1 对于模型 $(1.2)$, 给定一个初始数据 $u_{0} \in L^{1}\left(\mathbb{R} \cap L^{2}(\mathbb{R})\right)$, 存在 $\varepsilon>0, C\left(u_{0}\right)>0$, 使得对所 有的 $\left\|u_{0}\right\|_{L^{1}(\mathbb{R})}<\varepsilon$, 其存在唯一的全局解 $u \in C\left(\mathbb{R}_{+} ; L_{x}^{2}(\mathbb{R})\right) \cap C^{1}\left(\mathbb{R}_{+} ; H_{x}^{-2}(\mathbb{R})\right)$. 此外, $u$ 还满足

$$
t^{\frac{1}{2}}\|u(t)\|_{L_{x}^{\infty}(\mathbb{R})}+t^{\frac{1}{4}}\|u(t)\|_{L_{x}^{2}(\mathbb{R})} \leqslant C\left(u_{0}\right),
$$

且 $u$ 是不动点问题

$$
u(t, x)=K(t, \cdot) \star u_{0}+N * u^{2}
$$

的解, 其中 $K$ 和 $N$ 由

$$
K(t, x)=\frac{1}{2 \sqrt{\pi t}} \mathrm{e}^{-x^{-}} \mathrm{e}^{-\frac{x^{2}}{4 t}}\left(1+\frac{1}{2} \int_{0}^{+\infty} \mathrm{e}^{-\frac{\mu^{2}}{4 t}-\frac{\mu|x|}{2 t}-\frac{\mu}{2}} d \mu\right)
$$

和

$$
N(t, x)=\partial_{x}\left[\frac{1}{2 \sqrt{\pi t}} \mathrm{e}^{-x^{-}} \mathrm{e}^{-\frac{x^{2}}{4 t}}\left(1-\frac{1}{2} \int_{0}^{+\infty} \mathrm{e}^{-\frac{\mu^{2}}{4 t}-\frac{\mu|x|}{2 t}-\frac{\mu}{2}} d \mu\right)\right]
$$

给出, $x^{-}=\max (-x, 0), \star$ 表示通常空间上的卷积, 而 $*$ 是由

$$
v * w=\int_{0}^{t} \int_{\mathbb{R}} v(s, y) w(t-s, x-y) d y d s
$$

表示的时间 - 空间卷积, 这里假设此积分有意义.

该定理的证明读者可参见文献 [4].

本文考虑模型 (1.1) 和 Kakutani-Matsuuchi 模型 ${ }^{[5]}$ :

$$
u_{t}-\beta u_{t x x}+\nu\left(D^{\frac{1}{2}}+\mathcal{F}^{-1}\left(\mathrm{i}|\zeta|^{\frac{1}{2}} \operatorname{sign}(\zeta) \widehat{u}(\zeta)\right)\right)+\gamma u u_{x}=0,
$$

其中 $\nu$ 是黏性系数 (在原始的 Navier-Stokes 方程中, $\nu$ 是在某种归一化条件下的黏性系数), $\beta$ 和 $\gamma$ 分别是用来平衡几何色散和非线性项影响的参数. 本文所用到的 $D^{\frac{1}{2}}=(-\Delta)^{\frac{1}{2}}$ 为空间分数阶扩散算 子, $\widehat{u}(\zeta)$ 是 $u$ 的 Fourier 变换, $\mathcal{F}^{-1}(\cdot)$ 是 Fourier 逆变换. 在上面的方程中, 我们省略了 $u_{x}$ 项. 这是因 为我们考虑单向波, 因此, 我们可以作变换 $x^{\prime}=x+t, t^{\prime}=t$. 该模型描述了非局部黏性层的影响, 其非 局部项同时表现为扩散和色散. 通过线性色散分析, 此模型在很大程度上等价于文献 $[1,2]$ 中的模型. 其本身也与著名的 Ott-Sudan 模型 ${ }^{[6]}$ 类似, 带有非局部色散项.

这两个模型都含有非局部的分数阶导数项. 由于分数阶导数的全局性, 那么要求解该方程就显得 异常困难, 且其对存储的要求也非常高. Chen 等人 [4] 对该模型的等价积分形式采用了一个二阶的半隐 式差分格式. 而 Dumont 等人 ${ }^{[7]}$ 针对其原始模型运用 $\mathrm{GEAR}^{\alpha}{ }^{[8]}$ 格式进行离散. 对于第二类模型 (1.8), Chen 等人 ${ }^{[9]}$ 运用了一种半隐式的 Crank-Nicholson-leap-frog 守恒格式. 以上工作中的空间离散方法 都是基于有限差分的方法. 所以, 上述计算结果都是由低阶格式所完成的. 本文的一个主要目的是, 对 这两个模型构造高阶格式并用其进行数值模拟. 
由于对于这两种水波模型, 我们都可以假设其边界条件为周期边界条件. 因此, 我们就可用高精度 的 Fourier 谱方法进行空间离散. 因为这两个模型都含有非局部的分数阶导数项, 采用全局的 Fourier 谱方法离散和采用局部的有限差分方法运算量相当, 但精度大幅提高.

空间离散之后得到的是一个关于时间的常微分方程系统. 该系统一般既含线性项, 也含非线性项, 如果全部都是隐式求解的话, 代价将会极其昂贵. 因此, 我们采用半隐式格式来离散该系统. 一般的半 隐式格式很难达到高阶, 而谱缺陷校正法 ${ }^{[10]}$ 是一种求解常微分方程系统的稳定高阶算法. 为此, 本文 就将利用半隐式的谱缺陷校正法来离散这两类模型.

本文余下部分的结构安排如下: 第 2 节简单地介绍谱缺陷校正法和半隐式谱缺陷校正法; 第 3 节 对带时间分数阶导数的水波模型 (1.1) 进行数值模拟, 导出 Dias-Dutykh 模型解的衰减性质; 第 4 节 对 Kakutani-Matsuuchi 模型进行数值模拟并对其衰减性质进行研究; 最后一节进行总结.

下面首先给出衰减率的概念. 我们希望所得方程的解具有形式 $O\left(t^{a}\right)(a<0)$ 的衰减性质, 即当 $t$ 很大时, 有 $\|u(t, \cdot)\|_{L^{2}} \approx C t^{\alpha}$ 或者 $\|u(t, \cdot)\|_{L^{\infty}} \approx C t^{\alpha^{\prime}}$. 在数值上, 也就是说, 当 $t \rightarrow \infty$ 时, 以下的两种 比值:

$$
R_{2}=\frac{\log \left(\frac{\|u(t+\Delta t, \cdot)\|_{L^{2}}}{\|u(t, \cdot)\|_{L}{ }^{2}}\right)}{\log \left(\frac{t+\Delta t}{t}\right)} \quad \text { 和 } \quad R_{\infty}=\frac{\log \left(\frac{\|u(t+\Delta t, \cdot)\|_{L^{\infty}}}{\|u(t, \cdot)\|_{L}}\right)}{\log \left(\frac{(+\Delta t}{t}\right)}
$$

分别趋近于 $\alpha$ 和 $\alpha^{\prime}$. 其中 $\Delta t$ 表示时间步长. 之后都将用比值 $R_{2}$ 和 $R_{\infty}$ 来描述波的衰减率.

\section{2 谱缺陷校正法和半隐式谱缺陷校正法}

谱缺陷校正法 (SDC) 最早是由 Dutt 等人 ${ }^{[10]}$ 为求解常微分方程而提出的一种高阶稳定的数值方 法. 其基本思想就是, 将常微分方程化为等价的 Picard 积分方程, 然后将时间区间划分为一系列的子 区间，再在每个子区间上用 Gauss 积分进行离散. 具体做法就是, 首先用一种简单的时间离散格式对 方程进行求解, 然后在每个子区间上的 Gauss 型网格点上通过求解一系列的校正方程对解进行修正以 达到高阶精度.

接下来介绍对模型 (1.1) 的谱缺陷校正法. 空间由 Fourier 谱方法离散后, 我们将得到一个带分数 阶导数的常微分方程系统. 为简便起见, 考虑如下的单个常微分方程:

$$
\begin{aligned}
& \phi^{\prime}(t)+\frac{\sqrt{\nu}}{\sqrt{\pi}} \int_{0}^{t} \frac{\phi_{t}(s)}{\sqrt{t-s}} d s=F(t, \phi(t)), \quad t \in[0, T], \\
& \phi(0)=\phi_{0},
\end{aligned}
$$

其中 $\phi(t), \phi_{0} \in \mathbb{C}^{n}, F: \mathbb{R} \times \mathbb{C}^{n} \rightarrow \mathbb{C}^{n}$, 这里假定 $F$ 足够光滑以使得问题 (2.1) 是适定的.

将时间区域 $[0, T]$ 分成 $K$ 个非重叠的子区间: $[0, T]=\bigcup_{j=0}^{K-1}\left[t_{j}, t_{j+1}\right], t_{j}=j \Delta T$, 接下来将解 释谱缺陷校正法在区间 $\left[t_{j}, t_{j+1}\right]$ 上是如何求解的. 为了记号的方便, 我们用一般性区间 $[a, b]$ 来表示 $\left[t_{j}, t_{j+1}\right]$.

\section{1 校正方程}

谱缺陷校正法的首要关键点就是要将方程 (2.1) 写成等价的 Picard 积分方程

$$
\phi(t)=\phi_{a}+\int_{a}^{t} N_{0}(\nu(t-\tau)) F(\tau, \phi(\tau)) d \tau
$$


其中 $N_{0}(t)=\frac{1}{\sqrt{\pi}} \mathrm{e}^{t} \int_{t}^{+\infty} \frac{\mathrm{e}^{-s}}{\sqrt{s}} d s, t \geqslant 0$. 若方程 $(2.2)$ 有逼近解 $\phi^{0}(t)$, 定义其误差 $\delta(t)=\phi(t)-\phi^{0}(t)$. 另 一方面, 我们也可以用残差方程

$$
\epsilon\left(t, \phi^{0}\right)=\phi_{a}+\int_{a}^{t} N_{0}(\nu(t-\tau)) F\left(\tau, \phi^{0}(\tau)\right) d \tau-\phi^{0}(t)
$$

来衡量逼近解的性质. 由于 $\phi(t)=\phi^{0}(t)+\delta(t)$, 那么, 方程 (2.2) 可改写为

$$
\phi^{0}(t)+\delta(t)=\phi_{a}+\int_{a}^{t} N_{0}(\nu(t-\tau)) F\left(\tau, \phi^{0}(\tau)+\delta(\tau)\right) d \tau .
$$

联立该方程和方程 (2.3), 可以推导出方程

$$
\delta(t)=\int_{a}^{t} N_{0}(\nu(t-\tau))\left[F\left(\tau, \phi^{0}(\tau)+\delta(\tau)\right)-F\left(\tau, \phi^{0}(\tau)\right)\right] d \tau+\epsilon\left(t, \phi^{0}\right),
$$

这个就是我们所需要的校正方程.

\section{2 基于 Euler 方法的谱缺陷校正法}

首先用一列严格单增点列 $r_{1}, r_{2}, \ldots, r_{p}$ 来定义标准区间 $[-1,1]$ 上 $p$ 个 Legendre-Gauss-Radau IIa 点 (包含右端点), 用 $s_{1}, s_{2}, \ldots, s_{p}$ 来定义区间 $[a, b]$ 上 $p$ 个 Legendre-Gauss-Radau IIa 点, 易知, $r_{i}$ 与 $s_{i}, i=1,2, \ldots, p$ 之间有如下线性变换关系:

$$
s_{i}=\frac{b-a}{2} r_{i}+\frac{b+a}{2}, \quad i=1,2, \ldots, p .
$$

接着再根据 $[a, b]$ 上的 Legendre-Gauss-Radau IIa 点 $a=s_{0}<s_{1}<s_{2}<\ldots<s_{p}=b$, 将 $[a, b]$ 分成 $p$ 个子区间 $\left[s_{i}, s_{i+1}\right]$, 并定义 $\delta t_{i}=s_{i}-s_{i-1}$.

方程 $(2.2)$ 的初始解 $\phi^{0}\left(s_{i}\right)$ 可由某种特定有限差分离散 (如 Euler 方法) 得到. 现需要离散校正方 程 (2.4) 以计算出一系列校正项 $\delta_{i}^{k}$, 从而可得到一列精度逐渐提高的逼近解 $\phi^{k+1}=\phi^{k}+\delta^{k}$, 其中残差 方程 $\epsilon\left(s, \phi^{k}(s)\right)$ 可通过下式:

$$
\vec{\epsilon}=\vec{\phi}_{a}+\Delta T \boldsymbol{A} \vec{F}-\vec{\phi}^{k}
$$

近似求解, 其中 $\boldsymbol{A}$ 称为谱积分矩阵,

$$
A_{i j}=\frac{1}{2} \int_{-1}^{r_{i}} N_{0}\left(\frac{\Delta T}{2} \nu\left(r_{i}-s\right)\right) h_{j}(s) d s,
$$

其中 $h_{j}(s)$ 是基于标准区间 $[-1,1]$ 上 $p$ 个 Legendre-Gauss-Radau IIa 点的 Lagrange 插值多项式, 满 足 $h_{j}\left(x_{i}\right)=\delta_{i j}$. 当 $\nu=0$ 时, 该谱积分矩阵与文献 [11] 中的是一样的, 更详尽的描述可参见文献 [12].

现在用校正方程 (2.4) 来构造校正列 $\left\{\delta^{k}\right\}$ 和新的逼近解 $\left\{\phi^{k}\right\}$. 由方程 (2.4) 可以推出

$$
\begin{aligned}
\delta^{k}\left(s_{i+1}\right)= & \delta^{k}\left(s_{i}\right)+\int_{a}^{s_{i}}\left[N_{0}\left(\nu\left(s_{i+1}-\tau\right)\right)-N_{0}\left(\nu\left(s_{i}-\tau\right)\right)\right]\left[F\left(\tau, \phi^{k}(\tau)+\delta^{k}(\tau)\right)-F\left(\tau, \phi^{k}(\tau)\right)\right] d \tau \\
& +\int_{s_{i}}^{s_{i+1}} N_{0}\left(\nu\left(s_{i+1}-\tau\right)\right)\left[F\left(\tau, \phi^{k}(\tau)+\delta(\tau)\right)-F\left(\tau, \phi^{k}(\tau)\right)\right] d \tau+\epsilon_{i+1}\left(\phi^{k}\right)-\epsilon_{i}\left(\phi^{k}\right) .
\end{aligned}
$$

再根据向后 Euler 格式, 可得

$$
\delta_{i+1}^{k}=\delta_{i}^{k}+\sum_{l=1}^{i} \delta t_{l}\left[\left(N_{0}\left(\nu\left(s_{i+1}-s_{l}\right)\right)-N_{0}\left(\nu\left(s_{i}-s_{l}\right)\right)\right)\left(F\left(s_{l}, \phi_{l}^{k}+\delta_{l}^{k}\right)-F\left(s_{l}, \phi_{l}^{k}\right)\right)\right]
$$




$$
+\delta t_{i+1}\left[F\left(s_{i+1}, \phi_{i+1}^{k}+\delta_{i+1}^{k}\right)-F\left(s_{i+1}, \phi_{i+1}^{k}\right)\right]+\epsilon_{i+1}\left(\phi^{k}\right)-\epsilon_{i}\left(\phi^{k}\right) .
$$

另若将向后 Euler 格式换为向前 Euler 格式就可得到

$$
\begin{aligned}
\delta_{i+1}^{k}= & \delta_{i}^{k}+\sum_{l=1}^{i} \delta t_{l}\left[\left(N_{0}\left(\nu\left(s_{i+1}-s_{l}\right)\right)-N_{0}\left(\nu\left(s_{i}-s_{l}\right)\right)\right)\left(F\left(s_{l}, \phi_{l}^{k}+\delta_{l}^{k}\right)-F\left(s_{l}, \phi_{l}^{k}\right)\right)\right] \\
& +\delta t_{i+1} N_{0}\left(\nu \delta t_{i+1}\right)\left[F\left(s_{i}, \phi_{i}^{k}+\delta_{i}^{k}\right)-F\left(s_{i}, \phi_{i}^{k}\right)\right]+\epsilon_{i+1}\left(\phi^{k}\right)-\epsilon_{i}\left(\phi^{k}\right) .
\end{aligned}
$$

定义

$$
I_{i}^{i+1}\left(\phi^{k}\right)=\int_{a}^{s_{i}}\left[N_{0}\left(\nu\left(s_{i+1}-\tau\right)\right)-N_{0}\left(\nu\left(s_{i}-\tau\right)\right)\right] F\left(\tau, \phi^{k}(\tau)\right) d \tau+\int_{s_{i}}^{s_{i+1}} N_{0}\left(\nu\left(s_{i+1}-\tau\right)\right) F\left(\tau, \phi^{k}(\tau)\right) d \tau
$$

则由 (2.3) 可推导出

$$
I_{i}^{i+1}\left(\phi^{k}\right)=\epsilon_{i+1}\left(\phi^{k}\right)-\epsilon_{i}\left(\phi^{k}\right)+\phi_{i+1}^{k}-\phi_{i}^{k} .
$$

由于 $\phi_{l}^{k}+\delta_{l}^{k}=\phi_{l}^{k+1}, l=0,1, \ldots, p$, 将 (2.9) 和 (2.7) 两式相减可得

$$
\begin{aligned}
\phi_{i+1}^{k+1}= & \phi_{i}^{k+1}+I_{i}^{i+1}\left(\phi^{k}\right)+\sum_{l=1}^{i} \delta t_{l}\left[\left(N_{0}\left(\nu\left(s_{i+1}-s_{l}\right)\right)-N_{0}\left(\nu\left(s_{i}-s_{l}\right)\right)\right)\left(F\left(s_{l}, \phi_{l}^{k+1}\right)-F\left(s_{l}, \phi_{l}^{k}\right)\right)\right] \\
& +\delta t_{i+1}\left[F\left(s_{i+1}, \phi_{i+1}^{k+1}\right)-F\left(s_{i+1}, \phi_{i+1}^{k}\right)\right] .
\end{aligned}
$$

若将 $(2.9)$ 和 $(2.8)$ 两式相减, 则有

$$
\begin{aligned}
\phi_{i+1}^{k+1}= & \phi_{i}^{k+1}+I_{i}^{i+1}\left(\phi^{k}\right)+\sum_{l=1}^{i} \delta t_{l}\left[\left(N_{0}\left(\nu\left(s_{i+1}-s_{l}\right)\right)-N_{0}\left(\nu\left(s_{i}-s_{l}\right)\right)\right)\left(F\left(s_{l}, \phi_{l}^{k+1}\right)-F\left(s_{l}, \phi_{l}^{k}\right)\right)\right] \\
& +\delta t_{i+1} N_{0}\left(\nu \delta t_{i+1}\right)\left[F\left(s_{i}, \phi_{i}^{k+1}\right)-F\left(s_{i}, \phi_{i}^{k}\right)\right] .
\end{aligned}
$$

这样通过 $\phi^{k}$, 我们就可根据等式 (2.10) 或是 (2.11) 来计算新的逼近解 $\phi^{k+1}$. 现在还需特别注意的就 是怎么计算 $I_{i}^{i+1}\left(\phi^{k}\right)$, 而 $(2.9)$ 则给出了它的计算公式.

\section{3 半隐式谱缺陷校正格式}

通常情形下, 如我们前面所述, 单独使用向前或是向后 Euler 格式离散方程 (2.4) 不是一个好的选 择. 我们知道, 问题 (2.1) 中一般既含线性项也含非线性项, 因此, 将显示和隐式格式结合起来就更为 合理, 这就是我们所说的的半隐式的谱缺陷校正格式 (SISDC) (参见文献 [13-15]). 更确切地讲, 就是 将 $F(t, \phi(t))$ 分成两部分: $F_{I}(t, \phi(t))$ 和 $F_{E}(t, \phi(t))$, 其中 $F_{I}(t, \phi(t))$ 作隐式处理而 $F_{E}(t, \phi(t))$ 作显式 处理. 这样就得到了一种一阶的半隐式格式, 该格式可用来求解方程 $(2.2)$ 的初始逼近解 $\phi_{i}^{0}$. 类似地, 对于方程 (2.4), 其半隐式格式为

$$
\begin{aligned}
\delta_{i+1}^{k}= & \delta_{i}^{k}+\sum_{l=1}^{i} \delta t_{l}\left[\left(N_{0}\left(\nu\left(s_{i+1}-s_{l}\right)\right)-N_{0}\left(\nu\left(s_{i}-s_{l}\right)\right)\right)\left(F\left(s_{l}, \phi_{l}^{k}+\delta_{l}^{k}\right)-F\left(s_{l}, \phi_{l}^{k}\right)\right)\right] \\
& +\delta t_{i+1}\left[F_{I}\left(s_{i+1}, \phi_{i+1}^{k}+\delta_{i+1}^{k}\right)-F_{I}\left(s_{i+1}, \phi_{i+1}^{k}\right)+N_{0}\left(\nu \delta t_{i+1}\right)\left(F_{E}\left(s_{i}, \phi_{i}^{k}+\delta_{i}^{k}\right)-F_{E}\left(s_{i}, \phi_{i}^{k}\right)\right)\right] \\
& +\epsilon_{i+1}\left(\phi^{k}\right)-\epsilon_{i}\left(\phi^{k}\right),
\end{aligned}
$$


整理可得

$$
\begin{aligned}
\phi_{i+1}^{k+1}= & \phi_{i}^{k+1}+I_{i}^{i+1}\left(\phi^{k}\right)+\sum_{l=1}^{i} \delta t_{l}\left[\left(N_{0}\left(\nu\left(s_{i+1}-s_{l}\right)\right)-N_{0}\left(\nu\left(s_{i}-s_{l}\right)\right)\right)\left(F\left(s_{l}, \phi_{l}^{k+1}\right)-F\left(s_{l}, \phi_{l}^{k}\right)\right)\right] \\
& +\delta t_{i+1}\left[F_{I}\left(s_{i+1}, \phi_{i+1}^{k+1}\right)-F_{I}\left(s_{i+1}, \phi_{i+1}^{k}\right)+N_{0}\left(\nu \delta t_{i+1}\right)\left(F_{E}\left(s_{i}, \phi_{i}^{k+1}\right)-F_{E}\left(s_{i}, \phi_{i}^{k}\right)\right)\right] .
\end{aligned}
$$

总而言之, 对于给定的一个常微分方程系统 (2.1), 若要运用上述的半隐式谱缺陷校正法求解, 我 们只需要特别注明的就是如何将 $F$ 拆分为 $F_{I}$ 和 $F_{E}$. 一般情形下, 我们可取 $F_{I}$ 为线性部分, 而 $F_{E}$ 为非线性部分.

\section{3 对带黏性项的水波模型 (1.1) 衰减性质的数值研究}

\section{1 对水波模型 (1.1) 的半隐式谱缺陷校正法离散}

本节将研究带非局部黏性的 $\mathrm{KdV}$ 型方程 (1.1). 在数值计算中, 通过调节 $\alpha, \beta$ 和 $\gamma$ 的值, 我们可 以观察到方程各项, 即黏性扩散、几何色散和非线性项对波的影响.

首先进行空间离散. 为了获得好的逼近效果, 我们在实数域 $\mathbb{R}$ 上截取一长区间来进行模拟. 正如 我们在第 1 节中提到的一样, 空间上, 我们将采用标准的快速 Fourier 方法 (FFTW3.2) 进行离散. 我 们的目标就是希望能够精确模拟出波的传播过程. 给定波的初始值, 其将随着时间的推进而向右边界 传播, 我们希望我们的数值计算能够模拟出波向右端传播的物理过程.

将模型 (1.1) 进行 Fourier 变换可得

$$
\widehat{u}_{t}+\frac{\sqrt{\nu}}{\sqrt{\pi}} \int_{0}^{t} \frac{\widehat{u}_{t}(s)}{\sqrt{t-s}} d s=\widehat{f}=-\zeta^{2} \alpha \widehat{u}-\mathrm{i} \zeta \widehat{u}+\mathrm{i} \zeta^{3} \beta \widehat{u}-\mathrm{i} \zeta \gamma \mathcal{F}\left(\frac{u^{2}}{2}\right),
$$

其中 $\mathcal{F}$ 是 Fourier 变换算子. 上式的等价积分形式为

$$
\widehat{u}(t)=\widehat{u}_{0}+\int_{0}^{t} N_{0}(\nu(t-s)) F(s) d s,
$$

其中 $F=F_{I}+F_{E}, F_{I}=-\zeta^{2} \alpha \widehat{u}-\mathrm{i} \zeta \widehat{u}+\mathrm{i} \zeta^{3} \beta \widehat{u}, F_{E}=-\mathrm{i} \zeta \gamma \mathcal{F}\left(\frac{u^{2}}{2}\right)$. 我们有

$$
\begin{gathered}
\widehat{u}\left(t_{n+1}\right)=\widehat{u}\left(t_{n}\right)+\int_{0}^{t_{n}}\left(N_{0}\left(\nu\left(t_{n+1}-s\right)\right)-N\left(\nu\left(t_{n}-s\right)\right)\right) F(s) d s+\int_{t_{n}}^{t_{n+1}} N_{0}\left(\nu\left(t_{n+1}-s\right)\right) F(s) d s . \\
\text { 令 } \widehat{u}_{a}=\widehat{u}\left(t_{n}\right)+\int_{0}^{t_{n}}\left(N_{0}\left(\nu\left(t_{n+1}-s\right)\right)-N_{0}\left(\nu\left(t_{n}-s\right)\right)\right) F(s) d s, \text { 则方程 }(3.3) \text { 化为 } \\
\widehat{u}\left(t_{n+1}\right)=\widehat{u}_{a}+\int_{t_{n}}^{t_{n+1}} N_{0}\left(\nu\left(t_{n+1}-s\right)\right) F(s) d s .
\end{gathered}
$$

这样我们就可以运用前面所讲的半隐式谱缺陷校正法.

假设我们已得到每一个子区间 $\left[t_{k}, t_{k+1}\right], k=0,1, \ldots, n-1$ 上所有 Legendre-Gauss-Radau IIa 点 上的高精度解, 那么, 我们就可通过 Gauss Radau II 型积分得到 $\widehat{u}_{a}$, 即

$$
\widehat{u}_{a}=\widehat{u}^{n}+\sum_{k=1}^{n} \sum_{j=1}^{p}\left(N_{0}\left(\nu\left(t_{n+1}-s_{j}^{k}\right)\right)-N_{0}\left(\nu\left(t_{n}-s_{j}^{k}\right)\right)\right) F_{j}^{k} w_{j},
$$

其中 $s_{j}^{k}, j=1,2, \ldots, p$ 是对应区间 $\left[t_{k}, t_{k+1}\right], k=0,1, \ldots, n-1$ 上的 Legendre-Gauss-Radau IIa 点, $F_{j}^{k}$ 是 $F$ 在点 $s_{j}^{k}$ 上的对应的值, $w_{j}=\frac{\Delta T}{2} \bar{w}_{j}, j=1,2, \ldots, p$, 这里 $\bar{w}_{j}$ 是标准区间上的 Legendre-GaussRadau IIa 权. 


\section{2 数值结果}

现在来进行数值模拟, 本小节中所用的参数设置如下: 初始波 $u_{0}(x)=0.32 \operatorname{sech}^{2}\left(0.4\left(x-x_{0}\right)\right), x_{0}$ 为空间变量的中点, 空间计算区域设为 $[0,500]$, 那么, $x_{0}=250$, 时间步长为 $\Delta T=1$ 且 $p=8$, 空间步 长为 $h=0.2$. 对于 $\alpha=\nu=0, \beta=1, \gamma=6$, 初始波 $u_{0}(x)$ 会形成一个小振幅、长波的 $\mathrm{KdV}$ 孤立子.

首先给出两组参数下的数值解的误差来验证数值格式的收敛情形. 第一组参数为 $(\alpha, \nu, \beta, \gamma)=(5$, $1,0,0)$, 我们给出了该组数据下解在 $T=100$ 时刻下的 $L^{\infty}$ 和 $L^{2}$ 误差随着 $p$ 值增大的收玫情形, 如 图 1(a) 所示; 第二组参数为 $(\alpha, \nu, \beta, \gamma)=(0.1,0.1,1,6)$, 我们也同样给出了其在 $T=10$ 时刻两种误差 的收玫情形, 如图 1(b) 所示. 这两幅图都是以半对数坐标所画. 另外需要说明的就是, 由于在这两组 参数下我们无法给出其解析解, 因此, 我们以 $p=12$ 时的数值解作为精确解来求误差.

从图 1 可以看出, 该半隐式谱缺陷校正法对求带非局部黏性项的模型 (1.1) 的解是具有指数收 敛的.

图 2 为 $T=100$ 时刻的所得数值解. 图 2 中给出了在线性情形下 (即 $\gamma=0$ ), 对应于只非含局部 黏性项 $(\alpha, \nu)=(0,1)$ 、只含局部黏性项 $(\alpha, \nu)=(5,0)$ 和两项同时存在 $(\alpha, \nu)=(5,1)$ 时的波形图. 从 图 2 中可以观察到, 两种黏性项所产生的影响有很大不同.

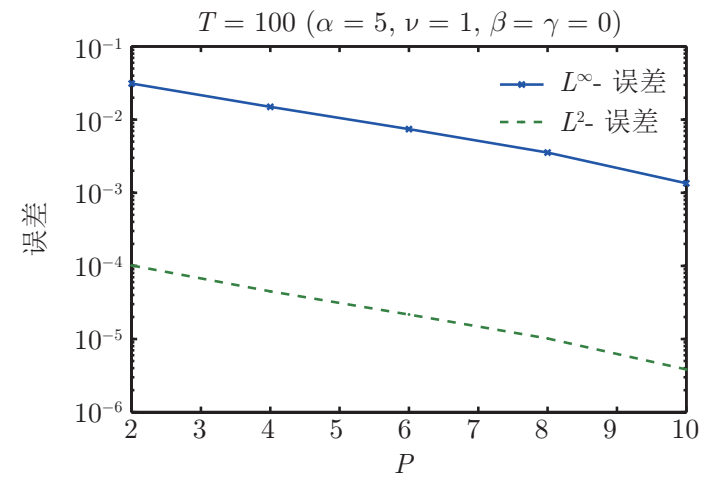

(a)

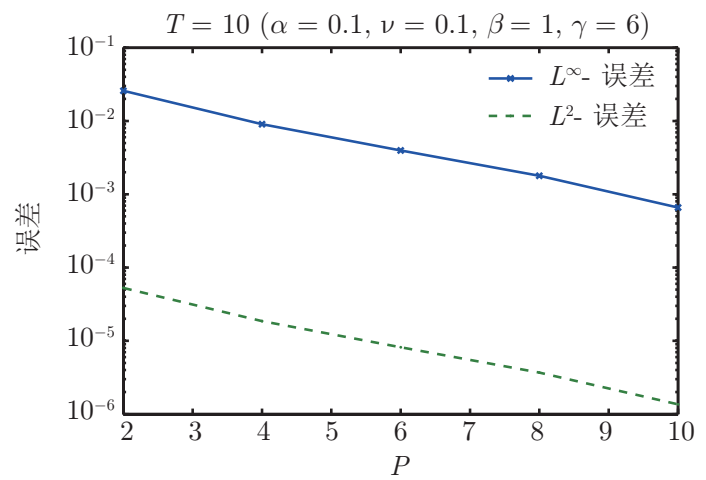

(b)

图 1 (a) $T=100$ 时误差 $(\alpha=5, \nu=1, \beta=\gamma=0)$; (b) $T=10$ 时误差 $(\alpha=0.1, \nu=0.1, \beta=1, \gamma=6)$

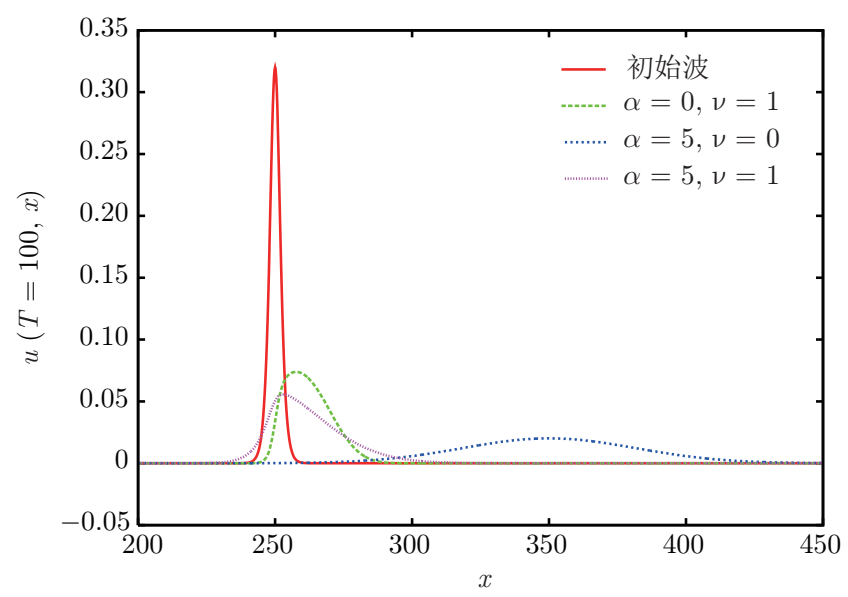

图 $2 T=100$ 时刻方程在不同黏性系数下的解 ( $\nu$ 等于 0 或 $1, \alpha$ 等于 0 或 $5, \gamma=\beta=0)$ 
注意到, 当 $(\alpha, \nu)=(0,0)$ 时, 初始波将以速度 1 向前传播. 所以, 在 $T=100$ 时, 该波将保持波形 向右传播且其中心为 $x=350$. 将其与 $(\alpha, \nu)=(0,1)$ 时相比较, 我们可以看到非局部黏性项极大地减 缓了波向前传播的速率, 还同时拉长了波长. 另一方面, 比较 $(\alpha, \nu)=(0,0)$ 和 $(\alpha, \nu)=(5,0)$ 两种情形 时, 我们发现局部的黏性项也拉长了波的波长, 不过其保持了波的传播速度. 当局部和非局部两种黏 性项同时作用时, 所得波会更接近只有非局部黏性项的情形.

接下来对定理 1 中关于方程解的衰减率的估计进行数值验证. 进一步地, 我们还将研究不包含在 定理 1 中的其他情形. 为此, 我们主要是研究黏性系数 $(\alpha, \nu)$ 分别为 $(0,0.1),(0.1,0),(0.1,0.1), \beta=0$, 以及 $\gamma$ 分别为 0 (线性) 和 1 (非线性) 时, 方程 $(1.1)$ 的解在区间 $(0,1000)$ 上 $L^{\infty}$ 和 $L^{2}$ 范数的衰减率.

根据在第 1 节中所述, 我们希望所求数值解具有形式为 $O\left(t^{a}\right), a<0$ 的衰减性质, 图 3(a) 和 3(b) 分别给出了线性情形 $(\gamma$ 为 0$)$ 下 $R_{\infty}$ 和 $R_{2}$ 随时间的变化.

从图 3(a) 中可以看出, 非局部的耗散项所导致的衰减要比局部耗散项所导致的要快. 所有的三种 情形的衰减率都是趋近于 0.5 的, 但其收玫都非常慢. 与 $L^{\infty}$ 一样, 我们同时也研究了 $L^{2}$ 范数的衰减 并得出了相类似的结论, 如图 3(b) 所示. 对于非线性情形, 即 $\gamma=1$ 时, 我们也对其进行了数值研究.

此外, 我们还根据在区间 $[T-200, T]$ 上所求得两种范数的数据, 通过最小二乘方法, 计算了它们 各自的衰减率, 我们将所得结果列在表 1 中. 这些结果与文献 [4, 定理 1.4] (即本文中定理 1) 中理论 结果相吻合. 同时还可以了解到对于线性和非线性情形, 其衰减情形差别不大.

如果将我们所算出的结果与文献 $[4,7]$ 中所得出的结果相比, 我们可以看出, 所有算出的这些衰减 率都只有 $10^{-2}$ 量级的误差. 由于半隐式谱缺陷校正是一个高阶格式, 我们的结果证实了文献 $[4,7]$ 中 的结果并给出了更精确的结果.

最后再来研究扩散和色散的影响. 现考虑方程 (1.1), 并将几何色散项 $u_{x x x}$ 考虑在内. 若不考虑黏 性项 $(\alpha=\nu=0)$, 则方程 (1.1) 的精确解为 $u(t, x)=u_{0}(x-1.64 t)$. 我们计算了方程 (1.1) 在几组不同 系数: $(\alpha, \nu, \beta, \gamma)=(0,0,1,6)$ (即 $\mathrm{KdV}$ 方程), $(\alpha, \nu, \beta, \gamma)=(0.1,0,1,6),(0,0.1,1,6),(0.1,0.1,1,6)$ 下的

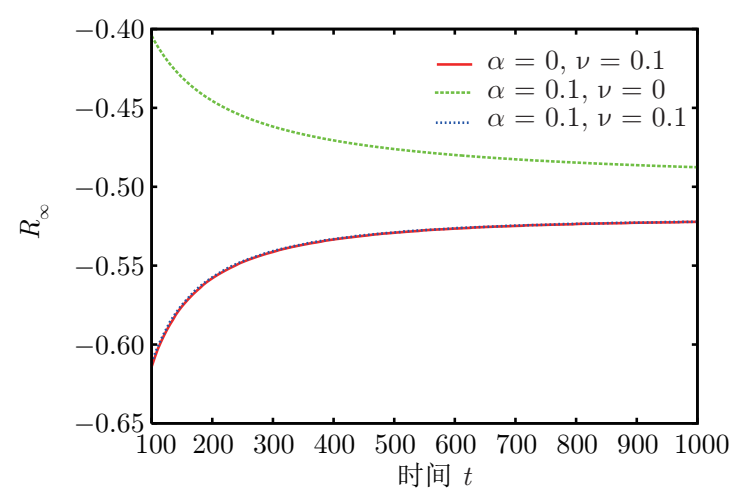

(a)

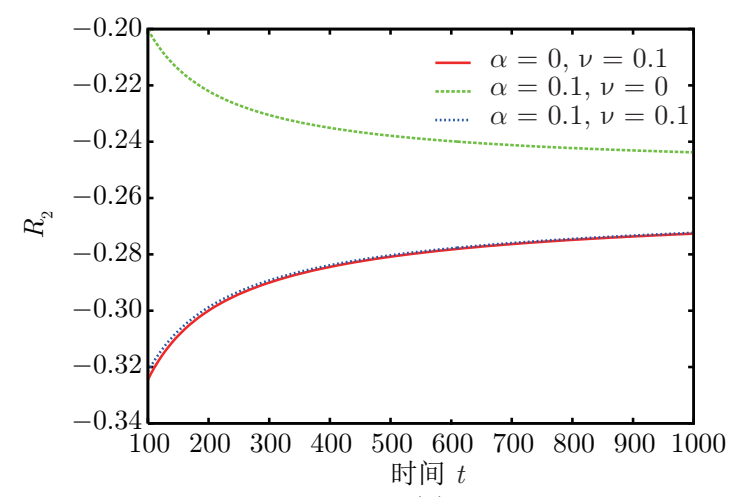

(b)

图 3 衰减率对比时间 $t(\nu$ 和 $\alpha$ 分别为 0 或 $0.1, \beta=\gamma=0)$. (a) $R_{\infty}$; (b) $R_{2}$

表 1 数值解 $u(t, \cdot)$ 的衰减率 $(\nu$ 和 $\alpha$ 分别为 0 或 $0.1, \beta=0, \gamma=0$ 或 1$)$

\begin{tabular}{ccccccc}
\hline 范数 & \multicolumn{2}{c}{$\alpha=0, \nu=0.1$} & \multicolumn{2}{c}{$\alpha=0.1, \nu=0$} & \multicolumn{2}{c}{$\alpha=0.1, \nu=0.1$} \\
& $\gamma=0$ & $\gamma=1$ & $\gamma=0$ & $\gamma=1$ & $\gamma=0$ & $\gamma=1$ \\
\hline$L^{\infty}$ & -0.5229 & -0.5189 & -0.4863 & -0.4930 & -0.5227 & -0.5188 \\
$L^{2}$ & -0.2736 & -0.2717 & -0.2431 & -0.2464 & -0.2733 & -0.2714 \\
\hline
\end{tabular}


解, 并将这些解和 $\mathrm{KdV}$ 方程的精确解描述在图 4 中作以比较. 同样可以看出, 局部耗散项减缓了波的 传播, 而局部的色散项则可能衍生出来双峰现象.

将图 4 与文献 [4, 图 5] 和 [7, 图 4] 相比, 我们观察出所有波形图整体上都具有相同的形状, 不过 我们所得出的波形图相比较于文献 [4, 图 5] 而言, 具有更明显的振荡, 这与文献 [7, 图 4] 类似.

现在再来研究在带有局部色散项情形下的衰减性质. 固定 $\beta=1, \gamma=6$, 我们来研究 $\alpha$ 和 $\nu$ 分别 为 0 和 1 时衰减情形. 所得结果如图 5 所示.

与表 1 类似, 我们也计算了上述情形下的衰减率, 如表 2 所示. 将这些结果与文献 $[4,7]$ 中所得结 果相比, 我们所得出的 $L^{\infty}$ 范数的衰减率更接近于 0.5 , 而 $L^{2}$ 范数衰减率更接近于 0.25 .

通过以上所有计算出的结果, 我们可知半隐式谱缺陷校正法所计算出的结果与文献 $[4,7]$ 中的相 吻合. 但是, 半隐式谱缺陷校正法是个高精度的数值算法, 其所算出的结果更为精确.

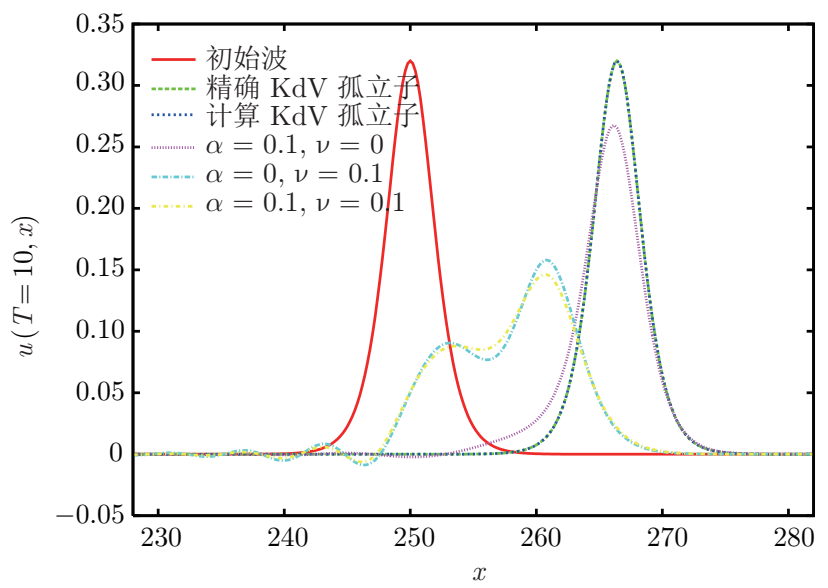

图 $4 T=10$ 时刻方程在不同黏性系数下的解 ( $\nu$ 和 $\alpha$ 分别为 0 或 $0.1, \beta=1, \gamma=6$ )

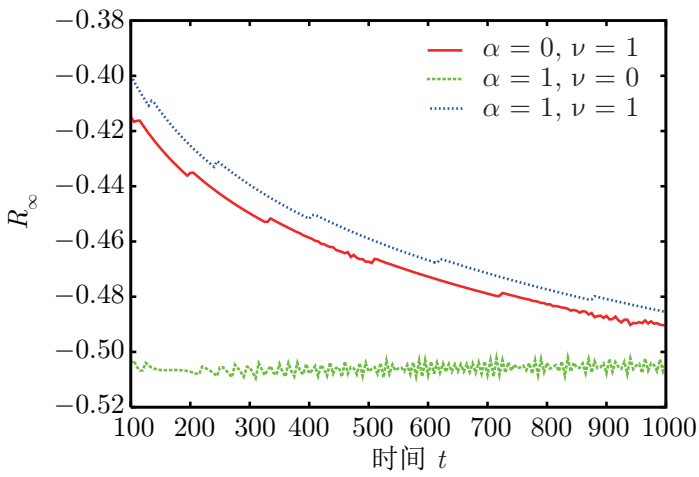

(a)

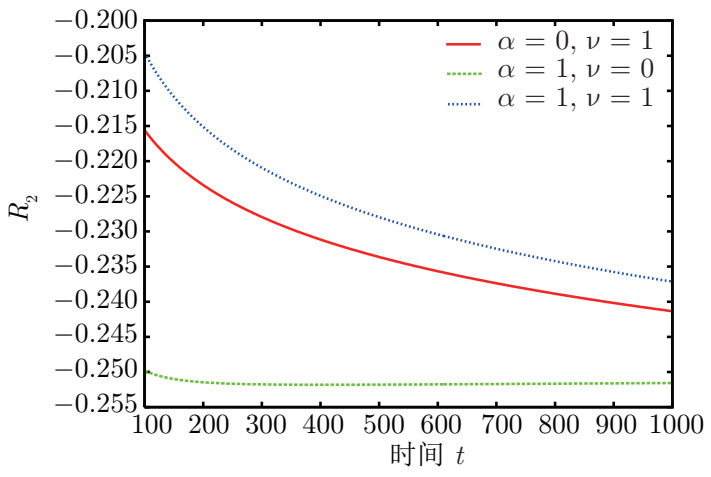

(b)

图 5 衰减率对比时间 $t(\nu$ 和 $\alpha$ 分别为 0 或 $1, \beta=1, \gamma=6)$. (a) $R_{\infty}$; (b) $R_{2}$

表 2 数值解 $u(t, \cdot)$ 的衰减率 ( $\nu$ 和 $\alpha$ 分别为 0 或 $1, \beta=1, \gamma=6$ )

\begin{tabular}{cccc}
\hline 范数 & $\alpha=0, \nu=1$ & $\alpha=1, \nu=0$ & $\alpha=1, \nu=1$ \\
\hline$L^{\infty}$ & -0.4874 & -0.5052 & -0.4814 \\
$L^{2}$ & -0.2402 & -0.2516 & -0.2358 \\
\hline
\end{tabular}




\section{4 对带空间分数阶扩散算子黏性项的水波模型衰减性质的数值研究}

本节要研究带空间分数阶扩散算子的水波模型 (Kakutani-Matsuuchi 模型) 的衰减性质.

\section{1 数值格式}

首先来简单介绍用来求解方程 (1.8) 的数值格式. 这里将 $u_{x}$ 项也放进计算过程中.

对于空间离散, 设计算区域长度为足够大的 $L$. 与第 3 节一样, 我们考虑周期边界条件, 并运用快 速 Fourier 变换. 对方程 (1.8) 进行 Fourier 变换可得其半离散格式为

$$
\widehat{u}_{t}=\frac{1}{\left(1+\beta \zeta^{2}\right)}\left(-\nu|\zeta|^{\frac{1}{2}}(1+\operatorname{isign}(\zeta)) \widehat{u}-\mathrm{i} \zeta\left(\widehat{u}+2 \gamma \mathcal{F}\left(\frac{u^{2}}{2}\right)\right)\right) .
$$

就上述方程而言, 其实际上就是常微分方程系统 (2.1) 在非局部项系数 $\nu=0$ 时的一个特殊情形, 其 Picard 积分形式为

$$
\widehat{u}_{t}=\widehat{u}_{0}+\frac{1}{\left(1+\beta \zeta^{2}\right)} \int_{0}^{t} F(s) d s,
$$

其中 $F=F_{I}+F_{E}, F_{I}=-\nu|\zeta|^{\frac{1}{2}}(1+\operatorname{isign}(\zeta)) \widehat{u}-\mathrm{i} \zeta \widehat{u}, F_{E}=-2 \mathrm{i} \zeta \gamma \mathcal{F}\left(\frac{u^{2}}{2}\right)$. 这样, 我们也就可以同样运用 半隐式谱缺陷校正法作时间离散.

\section{2 数值结果}

\subsection{1 数值格式的验证}

我们先来验证下数值格式的有效性. 根据文献 [16] 中的思想, 若将方程 (1.8) 中的黏性项省略, 那 么, 该方程具有如下形式的一族解析解,

$$
u_{e x}(t, x)=3 k \operatorname{sech}^{2}\left(c\left(x-x_{0}-(k+1) t\right)\right),
$$

其中 $c=\frac{1}{2} \sqrt{\frac{k}{k+1}}, k$ 和 $x_{0}$ 是任意常数 (参见文献 [17]). 然后就可以将数值结果与之进行对比以验证数 值格式的有效性. 设定计算区间长度 $L=5000$, 空间离散自由度为 10000 . 首先假设 $k=1, \Delta T=0.5$, $p=8$, 我们的计算值 $u(t, \cdot)$ 和精确值 $u_{e x}(t, \cdot)$ 在时间 $T=5$ 时刻的最大误差 $\left\|u_{e x}(T, \cdot)-u(T, \cdot)\right\|_{L^{\infty}}$ 能 很快达到 $O\left(10^{-10}\right)$ 量级的精度. 再设 $k=25, \Delta T=0.2, p=16$, 则算出 $\left\|u_{e x}(T, \cdot)-u(T, \cdot)\right\|_{L^{\infty}}$ 的值为 $1.09 \times 10^{-5}$. 这说明该数值格式是有效的且能达到很高精度.

\subsection{2 不同参数下的衰减率}

现在来研究参数 $\nu, \beta$ 和 $\gamma$ 对解的 $L^{2}$ 和 $L^{\infty}$ 两种范数衰减的影响, 类似地, 我们希望的衰减形式 为 $O\left(t^{\alpha}\right)$, 即当 $t$ 很大时, 有 $\|u(t, \cdot)\|_{L_{x}^{2}}=C t^{\alpha}$ 或者 $\|u(t, \cdot)\|_{L_{x}^{\infty}}=C t^{\alpha^{\prime}}$. 图 6 给出了 $\nu=0$ 时两种范数 衰减率 $R_{2}$ 和 $R_{\infty}$ 的变化趋势, 而图 7 则给出了 $\nu=1$ 时两种衰减率的变化趋势. 当时间 $t$ 趋近于无 穷时, $\alpha$ 和 $\alpha^{\prime}$ 应该要小于或等于 0 . 计算中, 空间区域给定为 $\Omega=[0,20000]$, 空间步长为 $h=2 \times 10^{-2}$, 时间步长为 $\Delta T=0.2, p=8$, 初始值为 $k=1$ 时的 BBM (Benjamin Bona Mahony) 孤立子方程 (4.3).

根据在区间 $[10,20]$ 上的数据, 运用最小二乘法, 我们计算出了各种参数下衰减率的值, 并将其列 入表 3 中. 从表 3 中可以得出, 当 $\nu=\beta=\gamma=0$ 时, 该波方程在 $L^{2}$ 和 $L^{\infty}$ 范数上都是守恒的. 而从 图 7 可以预测出, 当有黏性时 $(\nu=1), L^{2}$ 范数衰减率都为 -1 , 而 $L^{\infty}$ 范数衰减率为 -2 . 


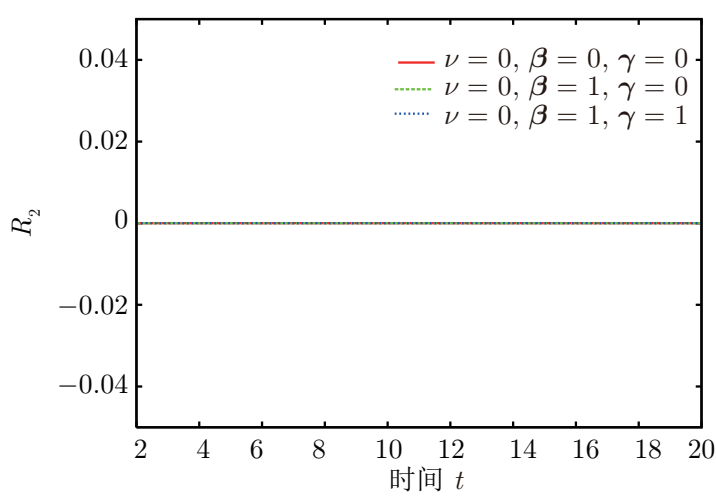

(a)

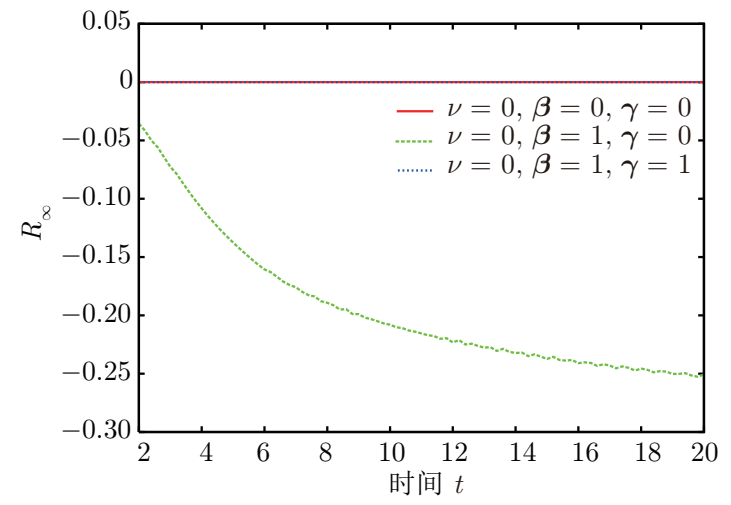

(b)

图 6 衰减率对比时间 $t(\nu=0, \beta, \gamma$ 等于 0 或 1$)$. (a) $R_{2}$, 三条曲线完全重叠; (b) $R_{\infty}$, 第一条与第三条曲线

\section{相互重叠}

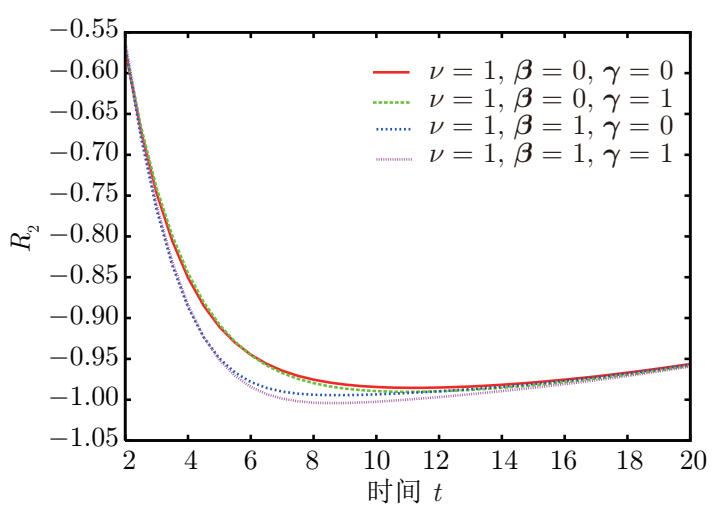

(a)

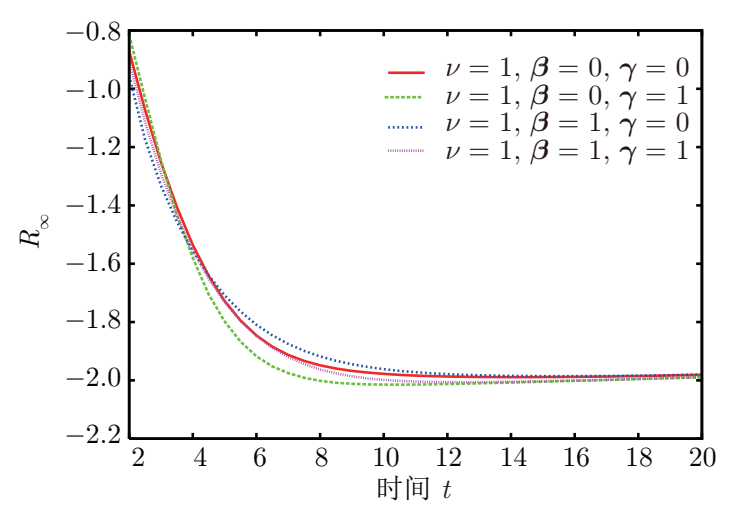

(b)

图 7 衰减率对比时间 $t(\nu=1, \beta, \gamma$ 等于 0 或 1$)$. (a) $R_{2}$, 衰减率趋近于 $-1 ;(\mathrm{b}) R_{\infty}$, 衰减率趋近于 -2

表 3 数值解 $u(t, \cdot)$ 的衰减率

\begin{tabular}{ccccc}
\hline 黏性 & 色散 & 非线性 & $L^{2}$ 衰减率 & $L^{\infty}$ 衰减率 \\
$\nu$ & $\beta$ & $\gamma$ & & \\
\hline 0 & 0 & 0 & 0.0000 & 0.0000 \\
0 & 1 & 1 & 0.0000 & 0.0000 \\
1 & 0 & 0 & -0.9772 & -1.9872 \\
1 & 0 & 1 & -0.9806 & -2.0042 \\
1 & 1 & 0 & -0.9794 & -1.9831 \\
1 & 1 & 1 & -0.9843 & -2.0015 \\
\hline
\end{tabular}

图 8 给出了 $t=7$ 时刻参数 $\nu, \beta$ 和 $\gamma$ 在不同值下的波形图. 从图 8 中可以看出, 当 $\beta=1$ 时, 波 出现了色散且在最大波峰左边有振荡现象. 而当 $\nu=1$ 时, 从图 $8(\mathrm{~b})$ 中可以看出波衰减非常快. 另外, 我们还可以了解到, 如果没有色散项, 则波对非线性项的依赖比较弱. 


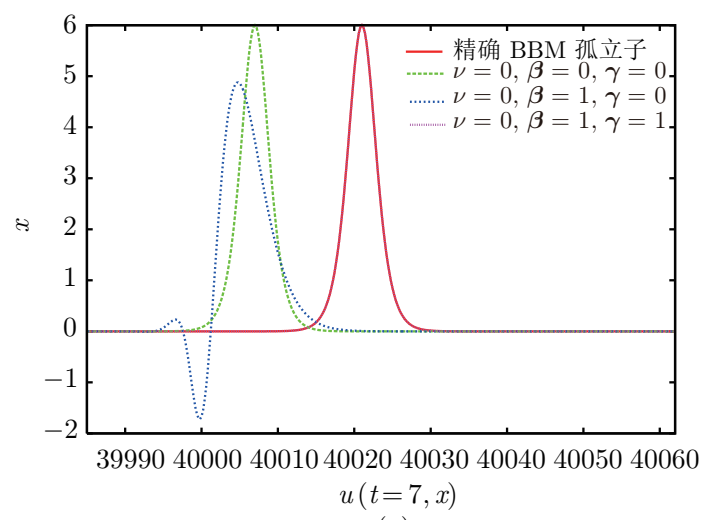

(a)

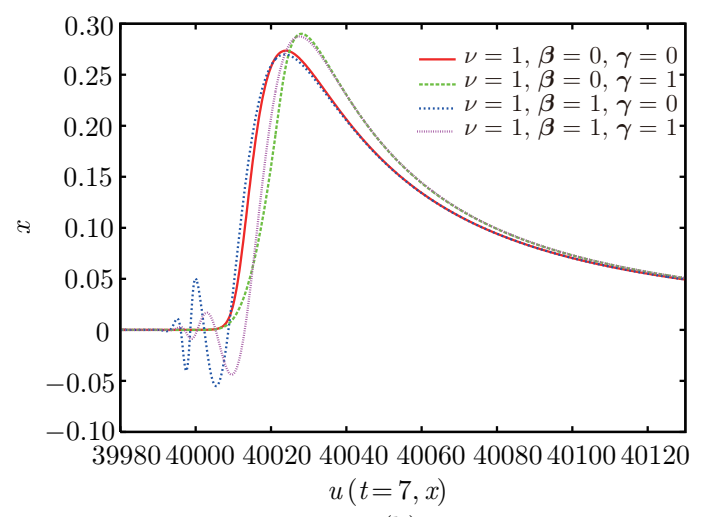

(b)

图 $8 t=7$ 时刻波形图 ( $\beta, \gamma$ 等于 0 或 1). (a) $\nu=0$, 第一条曲线 (精确的 BBM 孤立子) 和第四条曲线 (计 算的 BBM 孤立子) 相互重叠; (b) $\nu=1$

\subsection{3计算区间大小对衰减率的影响}

本节讨论计算区间的 $\Omega$ 的大小对波衰减率的影响. 图 9 给出了 $\Omega=[0, L], L$ 从 800 到 32000 时波 在两种范数下的衰减率. 其他参数的设置如下: 空间离散步长 $h$ 为 $2 \times 10^{-2}$, 时间离散步长为 $\Delta T=0.2$, $p=8$, 时间上的计算总长度为 $T=20$, 此外, $\nu=\beta=\gamma=1$. 从图 9 中可以看出, 在 $L<16000$ 时, 总 是捕捉不到稳定的衰减率.

\subsection{4 黏性对衰减率的影响}

接着来做一些数值实验来研究黏性对衰减的影响. 这里的数值计算所用的参数为 $L=16000, h=2$ $\times 10^{-2}, \Delta T=0.2, p=8, T=20, k=1, \beta=\gamma=1$.

从图 10 中可以看出, 如果黏性系数比较小 (如 $\nu$ 等于 0.1 ), 则需要比较长的时间才能得到一个好 的衰减率. 黏性系数越大, 波的衰减率就会收玫越快, 其中 $L^{2}$ 范数衰减率会收玫到 -1 , 而 $L^{\infty}$ 范数衰 减率收玫到 -2 . 另外, 我们也看到, 正如前面所讨论的计算区间的影响, 随着时间继续推进, 这些衰减 率就会开始减小, 而且 $\nu$ 越大, 开始减小的时间就越早. 图 11(a) 给出了方程 (1.8) 在不同黏性系数 $\nu$ 下的解. 从图 11(a) 中容易观察到, $\nu$ 越大, 波下降越明显, 且其传播也更快.

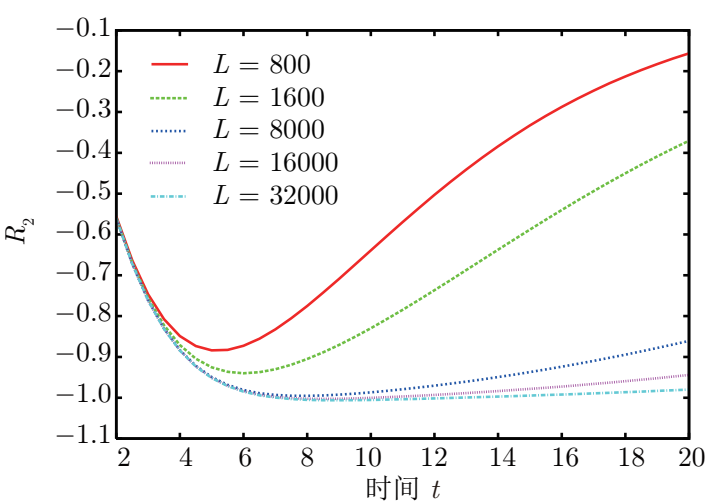

(a)

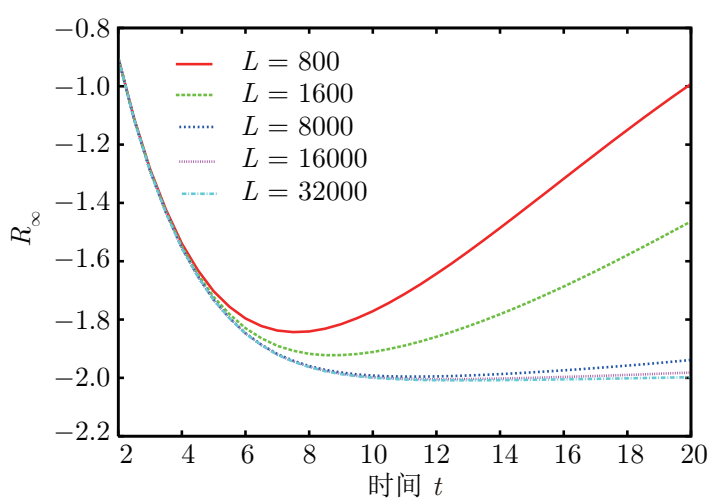

(b)

图 9 衰减率对比时间 $t, t=[0, L], L$ 从 800 到 $32000(\nu=\beta=\gamma=1)$. (a) $R_{2} ;(b) R_{\infty}$ 


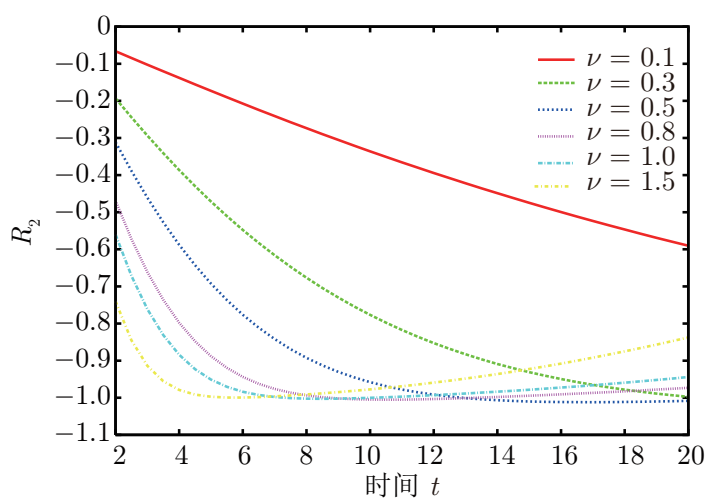

(a)

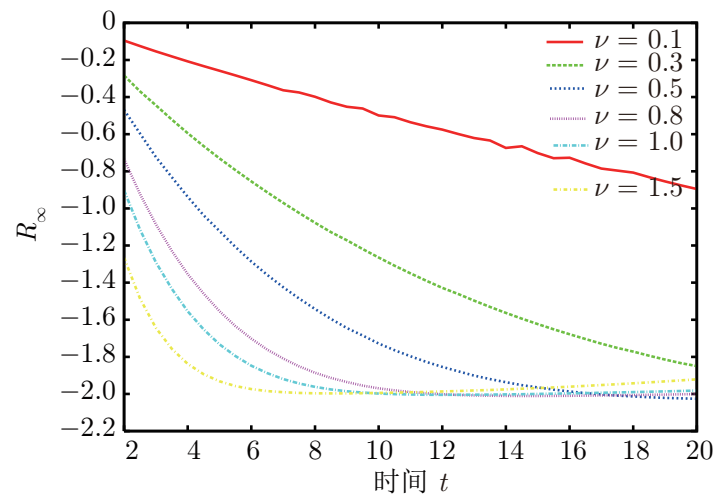

(b)

图 10 不同黏性系数下衰减率与时间 $t$ 的对比, $\nu$ 从 0.1 到 1.5. (a) $R_{2} ;$ (b) $R_{\infty}$

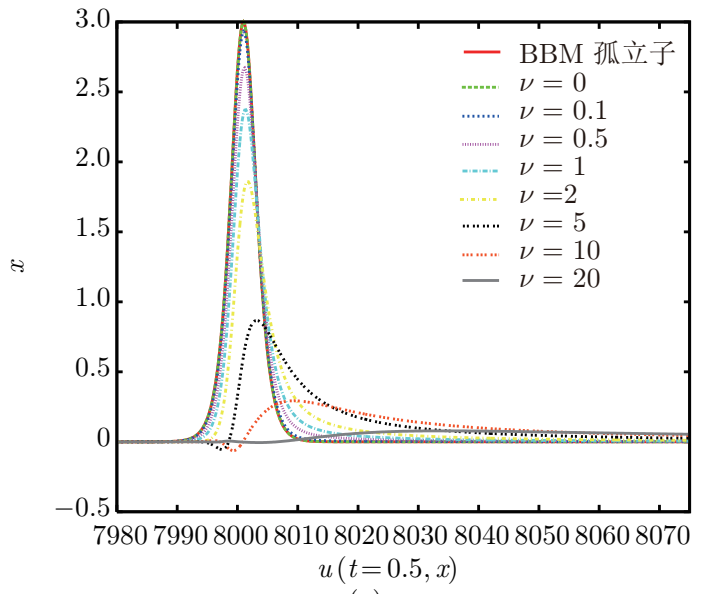

(a)

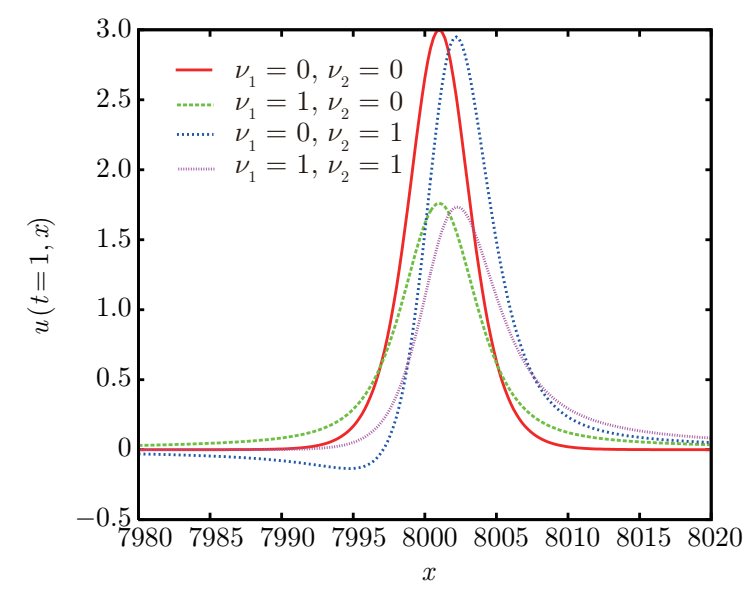

(b)

图 11 (a) 不同黏性系数下的波形图, $\nu$ 从 0 到 $20 ;(\mathrm{b}) \nu_{1}$ 和 $\nu_{2}$ 不同时的波形图 $\left(\nu_{1}\right.$ 和 $\nu_{2}$ 分别为 0 和 1)

\subsection{5 不同黍性项对衰减率的影响}

最后将方程 (1.8) 中的黏性项分为如下的两部分:

$$
G(u)=\nu_{1} D^{\frac{1}{2}}+\nu_{2} \mathcal{F}^{-1}\left(\mathrm{i}|\zeta|^{\frac{1}{2}} \operatorname{sign}(\zeta) \widehat{u}(\zeta)\right),
$$

并讨论它们两者之间对波衰减率的影响. 图 11(b) 给出的是在 $T=1$ 时刻, $\beta=\gamma=0$ 时的波形图, 初 始值 $u_{0}$ 为 BBM 孤立子, $h=2 \times 10^{-2}, \Delta T=0.2, p=8$, 黏性系数 $\nu_{1}$ 和 $\nu_{2}$ 各自分别为 0 或 1 .

图 12 说明了, 当 $\nu_{1}=\nu_{2}=0$ 时, 方程 (1.8) 不管是在 $L^{2}$ 范数还是在 $L^{\infty}$ 范数意义下都是守恒的, 当 $\nu_{1}=1, \nu_{2}=0$ 时, 方程只有扩散; 而当 $\nu_{1}=0, \nu_{2}=1$ 时, 方程则只有色散; 若 $\nu_{1}$ 和 $\nu_{2}$ 同时为 1 , 则扩散和色散都会出现. 另外, 我们注意到, 从图 12(b) 第三条曲线了解到, 在 $\nu_{1}=0$ 和 $\nu_{2}=1$ 时波 的 $L_{\infty}$ 范数并不是一直都在衰减, 而是在开始衰减过程中, 其衰减速率越来越小, 直到为零后开始增 长, 增长速率也是越来越小, 然后又开始衰减, 如此交替进行. 为了了解在此参数下波的传播状况, 我 们在图 13 中给出了其不同时刻下的波形图. 从图 13 中可以观察到, 随着时间的推进, 波的上下两个 波峰彼消此长. 


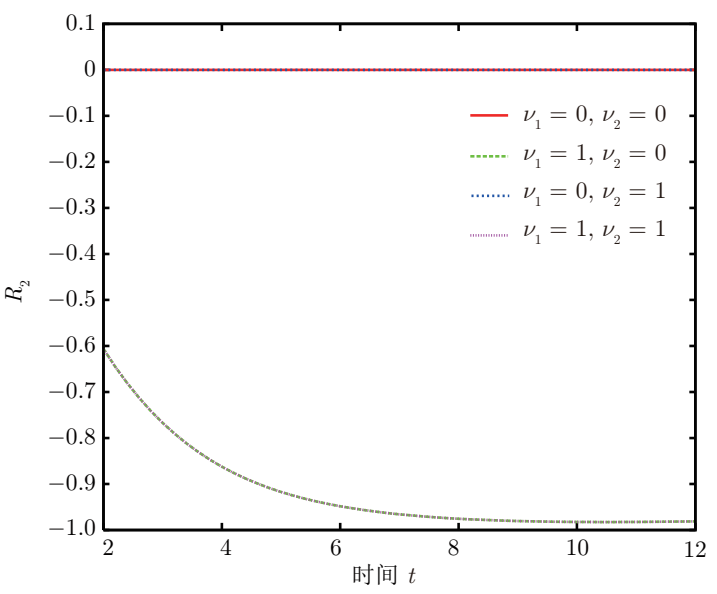

(a)

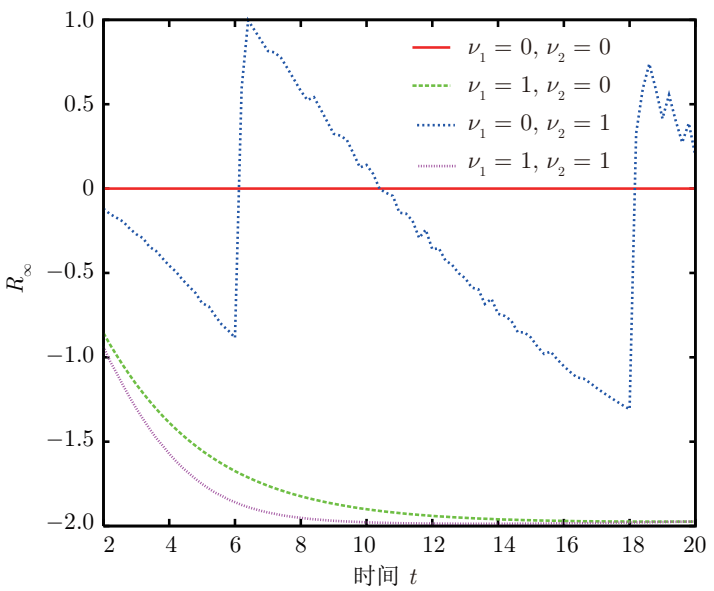

(b)

图 $12 \nu_{1}$ 和 $\nu_{2}$ 不同时衰减率对比时间 $t\left(\nu_{1}\right.$ 和 $\nu_{2}$ 分别为 0 或 1$)$. (a) $R_{2}$, 第一条和第三条曲线相互重叠, 第 二条和第四条曲线相互重叠, (b) $R_{\infty}$

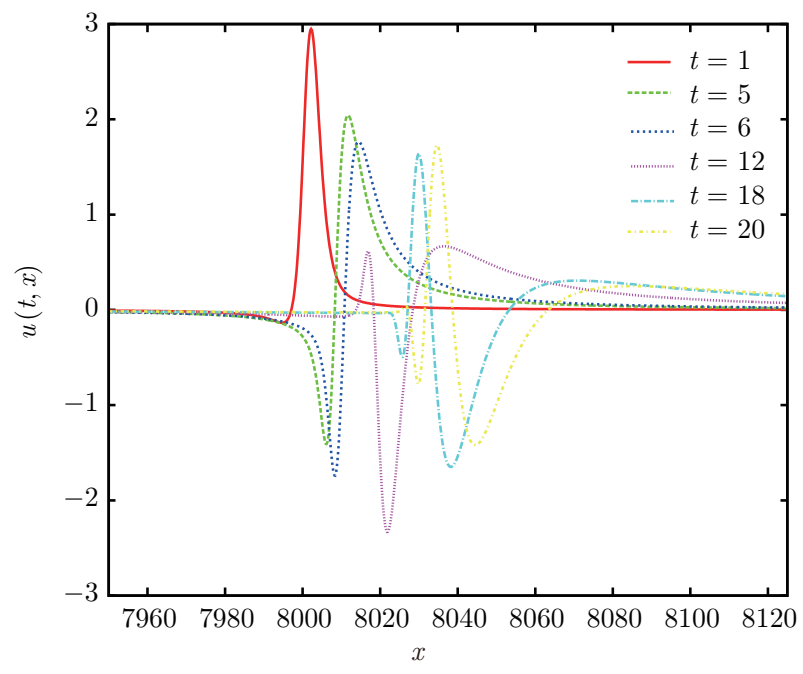

图 $13 \nu_{1}=0$ 和 $\nu_{2}=1$ 时波在不同时刻的波形图

\section{5 结论}

本文主要研究了两类具有周期边界的带非局部黏性项水波模型随着时间推进的衰减性质,第一类 模型为 $\mathrm{KdV}$ 型模型, 其非局部黏性表现为时间 $\frac{1}{2}$ 阶导数, 而第二类模型是非线性黏性表现为空间分 数阶算子形式的 Kakutani-Matsuuchi 模型.

首先通过 Fourier 变换, 将上述两种模型进行空间离散, 再对所得常微分系统转化成等价的 Picard 积分方程, 进一步采用半隐式的谱缺陷校正法模拟该两类波随时间的传播过程, 并通过大量数值试验 对这两类带分数阶导数项水波模型的衰减性质进行数值研究. 通过调节方程 (1.1) 和 (1.8) 中不同参数 的值, 本文研究了黏性项、几何色散项、非线性项和计算区域大小对模型衰减性质的影响. 数值模拟过 
程中所采用的半隐式谱缺陷校正法对所得常微分方程系统的线性部分作隐式处理, 对非线性部分作显 示处理, 充分利用了半隐式格式的计算方便性和谱缺陷校正法的高阶性质. 所得实验结果与已有理论 相吻合, 并提供了更为精确的计算.

\section{参考文献}

1 Liu P L F, Orfila A. Viscous effects on transient long-wave propagation. J Fluid Mech, 2004, 520: 83-92

2 Dutykh D, Dias F. Viscous potential free-surface flows in a fluid layer of finite depth. Comput Rendus Math, 2007, 345: $113-118$

3 Dutykh D. Visco-potential free-surface flows and long wave modelling. Eur J Mech B Fluids, 2009, 28: 430-443

4 Chen M, Dumont S, Dupaigne L, et al. Decay of solutions to a water wave model with a nonlocal viscous dispersive term. Discrete Contin Dyn Syst, 2010, 27: 1473-1492

5 Kakutani T, Matsuuchi K. Effect of viscosity on long gravity waves. J Phys Soc Japan, 1975, 39: 237-246

6 Ott E, Sudan R. Damping of solitary waves. Phys Fluids, 1970, 13: 14-32

7 Dumont S, Duval J. Applications of the gear scheme for the numerical study of the decay of solutions to viscous water wave models. Int J Numer Anal Model, 2013, 10: 333-349

8 Galucio A C, Deü J F, Mengué S, et al. An adaptation of the Gear scheme for fractional derivatives. Comput Methods Appl Mech Engrg, 2006, 195: 6073-6085

9 Chen M, Dumont S, Goubet O. Decay of solutions to a viscous asymptotical model for waterwaves: Kakutanimatsuuchi model. Nonlinear Anal, 2012, 75: 2883-2896

10 Dutt A, Greengard L, Rokhlin V. Spectral deferred correction methods for ordinary differential equations. BIT, 2000, 40: $241-266$

11 Greengard L. Spectral integration and two-point boundary value problems. SIAM J Numer Anal, 1991, 28: 1071-1080

12 Huang J, Jia J, Minion M. Accelerating the convergence of spectral deferred correction methods. J Comput Phys, 2006, 214: 633-656

13 Minion M L. Semi-implicit spectral deferred correction methods for ordinary differential equations. Commun Math Sci, 2003, 1: 471-500

14 Minion M L. Semi-implicit projection methods for incompressible flow based on spectral deferred corrections. Appl Numer Math, 2004, 48: 369-387

15 Liu F, Shen J. Stabilized semi-implicit spectral deferred correction methods for allen-cahn and cahn-hilliard equations. Math Methods Appl Sci, doi: 10.1002/mma.2869, 2013

16 Chen M. Numerical investigation of a two-dimensional boussinesq system. Discrete Contin Dyn Syst, 2009, 23: 11691190

17 Eilbeck J, McGuire G. Numerical study of the regularized long-wave equation I: Numerical methods. J Comput Phys, 1975, 19: 43-57

\section{A semi-implicit spectral deferred correction method for two water wave models with nonlocal viscous term and numerical study of their decay rates}

\section{MAO ZhiPing \& SHEN Jie}

Abstract In this paper, we numerically investigate the decay rates of two nonlocal water wave models. One is in the $\mathrm{KdV}$ form with a time nonlocal viscous dispersive term

$$
u_{t}+u_{x}+\beta u_{x x x}+\frac{\sqrt{\nu}}{\sqrt{\pi}} \int_{0}^{t} \frac{u_{t}(s)}{\sqrt{t-s}} d s+u u_{x}=\nu u_{x x}
$$


Another is the spatial nonlocal viscous diffusion-dispersive Kakutani-Matsuuchi model

$$
u_{t}-\beta u_{t x x}+\nu\left(D^{\frac{1}{2}}+\mathcal{F}^{-1}\left(\mathrm{i}|\zeta|^{\frac{1}{2}} \operatorname{sign}(\zeta) \widehat{u}(\zeta)\right)\right)+\gamma u u_{x}=0 .
$$

For the discretization of these two models, we use Fourier method in spatial, while in temporal, with the help of computational conveniences of semi-implicit and high order property of spectral defect correction, we a semiimplicit spectral defect correction (SISDC) method. Then we study the influences of viscous, geometric dispersion, nonlinearity and computational domain size by plenty of numerical experiment.

Keywords water wave, nonlocal, decay rate, semi-implicit, spectral deferred correction

MSC(2010) 35Q35, 35Q53, 76B15

doi: $10.1360 /$ N012014-00225 